\title{
Indonesian women's views on health providers' nutrition advice during pregnancy
}

\author{
W. Rahmawati ${ }^{1,2}$, J.C. Willcox ${ }^{3}$, P. van der Pligt ${ }^{1}$ and A. Worsley ${ }^{1}$ \\ ${ }^{1}$ School of Exercise and Nutrition Sciences, Deakin University, Burwood 3125, Victoria, Australia, \\ ${ }^{2}$ Department of Nutrition Science, Faculty of Medicine, Universitas Brawijaya, Malang 65145, East Java, Indonesia \\ and \\ ${ }^{3}$ School of Allied Health, College of Science, Health and Engineering, La Trobe University, Bundoora 3083, \\ Victoria, Australia
}

Promoting healthy nutrition during pregnancy is crucial for the optimal health of both mother and infants in Indonesia where maternal Iron Deficiency Anaemia (IDA) (37\%) contributes to the high prevalence of low birth weight (10.2\%), children's IDA (28\%) and stunting (34.5\%). Understanding women's experiences in obtaining nutritional information from antenatal health professionals (HPs) is crucial to plan better nutrition education. However, there have been few studies about Indonesian women's experiences of antenatal nutrition education. The purpose of this qualitative enquiry was to investigate Indonesian women's views of HPs' nutrition advice during their pregnancies.

Semi-structured interviews were conducted with 23 pregnant women in Malang City, Indonesia, and the women's responses were thematically analysed.

We found three key themes. Theme 1: Trust. Half of women $(n=11 / 23)$ reported trusting nutrition advice from HPs. A few women believed that HPs were beneficial, made maximum efforts to provide nutrition education. A couple of women reported trusting HPs because they viewed that HPs know more than their family. However, two women disclosed that they could not apply the HPs' nutrition advice in their daily lives. A few women also vocalised that women needed to be pro-active and report their problems to HPs. Theme 2: Adequate information. Only a small number of women reported that HPs' nutrition advice met their needs. While some women suggested that the nutrition advice was sufficient, twice as many viewed the information as inadequate. Of the women viewing the information as adequate, a few stated that the HPs information was adequate because they didn't rely on the HPs information as their only source. Those women sought information from other sources, including their mother or mother-in-law, social media or the internet A small number of women expressed that they were not sure what antenatal nutrition information they required. Many women revealed that HPs focused on pregnancy health problems, program achievement, medical treatment or supplements rather than dietary advice. Theme 3: Criticisms. Many women recounted that it required work on their behalf to obtain antenatal nutrition information. Some reported that they needed to prepare questions if they wanted an explanation from the HPs, while others revealed that the limited time during consultations made it difficult to ask questions. In addition, some disclosed that it was easier to search online rather than to ask the HPs.

In conclusion, HPs were seen to provide adequate nutrition education by less than half of the study participants. However, further research is required to map antenatal nutrition education programs and information sources in Indonesia to meet women's needs. A consistent nutrition information provided by the different types of Indonesian health professionals and a combined nutrition education approach is necessary.

1. Girard AW \& Olude O (2012) Paediatr Perinat Epidemiol 26, 191-204.

2. Bookari K, Yeatman H \& Williamson M (2016) Int $J$ Womens Health 2, 405-419.

3. Rahmawati W, Willcox JC, van der Pligt P \& Worsley A (2020) Midwifery, Under review.

4. World Bank Group (2018) 